\section{Cahiers de Narratologie}

Analyse et théorie narratives

14 | 2008

Prose d'idées : formes et savoirs

\title{
Réflexions sur le texte (pseudo-)littéraire chez Paolo Mantegazza : Un giorno a Madera. Una pagina dell'igiene d'amore
}

\section{Edwige Comoy Fusaro}

\section{OpenEdition}

Journals

Édition électronique

URL : http://journals.openedition.org/narratologie/543

DOI : $10.4000 /$ narratologie.543

ISSN : 1765-307X

Éditeur

LIRCES

Référence électronique

Edwige Comoy Fusaro, « Réflexions sur le texte (pseudo-)littéraire chez Paolo Mantegazza : Un giorno a Madera. Una pagina dell'igiene d'amore », Cahiers de Narratologie [En ligne], 14 | 2008, mis en ligne le 05 janvier 2011, consulté le 19 avril 2019. URL : http://journals.openedition.org/narratologie/543 ; DOI : $10.4000 /$ narratologie.543

Ce document a été généré automatiquement le 19 avril 2019

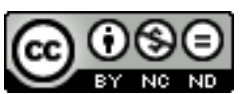

Cahiers de Narratologie - Analyse et théorie narratives est mis à disposition selon les termes de la licence Creative Commons Attribution - Pas d'Utilisation Commerciale - Pas de Modification 4.0 International. 


\title{
Réflexions sur le texte (pseudo- )littéraire chez Paolo Mantegazza : Un giorno a Madera. Una pagina dell'igiene d'amore
}

\author{
Edwige Comoy Fusaro
}

1 Paolo Mantegazza est l'un des hommes publics italiens qui ont le plus marqué leur temps. Médecin lombard né en 1831, il s'est moins illustré par ses recherches scientifiques que par son activité parallèle d'écrivain, au sens le plus large possible : c'est le précurseur et le parangon du divulgateur scientifique de la deuxième moitié du XIX ${ }^{e}$ siècle. Sa prose, riche et variée, est difficilement classable parce que des éléments de différente nature s'y mêlent inextricablement, notamment la littérature et la médecine. D’ailleurs, la question du genre s'est systématiquement posée à quiconque aborde les textes de Mantegazza ${ }^{1}$. Les quelques romans de son vaste corpus peuvent être rangés, faute de mieux, sous l'appellation vague de "pseudo-littérature ", mais peut-être la catégorie de la prose d'idées convient-elle davantage à cette production sui generis. C'est ce que nous allons nous efforcer de tirer au clair en analysant l'hybridisme générique de son premier roman, Un giorno a Madera. Una pagina dell'igiene d'amore ${ }^{2}$.

2 L'hétérogénéité des œuvres de Mantegazza et son engagement personnel dans les causes qu'il défend sont le signe d'une caractéristique encore humaniste, au sens large, des savants du XIXe siècle. Il agit pourtant au nom du modernisme puisque son arme de combat est le savoir scientifique qui est, à l'époque, en constante évolution, ce qui suppose qu'il est nécessairement à la pointe du progrès. Ces deux aspects apparemment contradictoires s'harmonisent à la lumière du contexte très particulier des années du positivisme en Italie, c'est-à-dire entre l'Unité et 1900, l'époque du passage du règne des intellectuels éclairés (comme Vieusseux) à celui des capitaines d'industrie. La science positiviste s'affirme alors comme la religion des temps modernes et les élites scientifiques jouissent d'une position prodigieusement privilégiée. Dotés d'un prestige et d'une autorité incontestés, quelques savants comme Mantegazza et, dans une moindre mesure, 
Lombroso, encouragés par l'immense succès que remportent leurs œuvres, s'arrogent une sorte de licence d'omniscience en vertu de laquelle ils interviennent dans toute sorte de domaines plus ou moins annexes à leur domaine de spécialité - la jurisprudence, la politique, l'éducation, la littérature et les arts, la sexualité, la cuisine ou encore le spiritisme (la liste n'est pas exhaustive). Cette mainmise sur la globalité des activités humaine implique un débordement des champs de compétence : de fait, dans les œuvres qui ne s'adressent pas aux spécialistes, le discours scientifique est relégué au rang de prétexte et la parfaite objectivité de l'homme de science positiviste cède le pas à l'opinion subjective érigée en règle de vie. Plus que de simples savants, ils deviennent les vecteurs et les promoteurs d'un système de pensée, d'une morale, d'une idéologie. Mantegazza, surtout, auteur d'œuvres à la fois pseudo-littéraires et pseudo-scientifiques, est à cet égard le champion du paternalisme éclairé des élites intellectuelles de la fin du XIX siècle.

3 En marge de ses recherches expérimentales de laboratoire, Mantegazza manifeste dès l'âge de vingt-trois ans son désir d'aborder l'homme dans sa totalité, en dépassant les clivages instaurés par la spécialisation de plus en plus accrue des savoirs. Il trouve son domaine de prédilection dans l'anthropologie, qu'il conçoit comme une synthèse d'anatomie, de physiologie, de psychologie et de métaphysique, autrement dit comme "l'histoire naturelle de l'homme " ${ }^{3}$. Sa thèse de médecine porte sur la Physiologie du plaisir $(1854)^{4}$. Déjà les sciences médicales côtoient les sciences humaines ${ }^{5}$. Sa vocation d'anthropologue se confirme lors de son séjour de quatre ans en Argentine et en Uruguay. L'exercice de la médecine à Buenos Aires lui permet d'étudier les populations d'Amérique latine et il en tire des observations ethnologiques publiées à son retour en Italie. En 1860 il gagne le concours qui lui permet d'accéder à la chaire de Pathologie générale à l'université de Pavie ${ }^{6}$ puis, en 1869, il quitte Pavie pour enseigner l'anthropologie et l'ethnologie à l'Institut d'Etudes Supérieures de Florence. Il ne cessera jamais d'enseigner jusqu'à sa mort, en 1910.

4 Au-delà de sa formation initiale de médecin, Mantegazza se spécialise ainsi dans différents domaines scientifiques: il est physiologiste, anthropologue, ethnologue, pathologiste, puis hygiéniste et hydrologue médical. Il mène également des travaux en histologie $^{7}$, puis en psychologie ${ }^{8}$, en esthétisme et physiognomonie ${ }^{9}$ et, dès le début de sa carrière scientifique, il est l'un des disciples et propagateurs de l'évolutionnisme darwinien ${ }^{10}$. C'est à Pavie qu'il s'essaie pour la première fois à la littérature populaire avec la publication de Il bene e il male. Libro per tutti (1861) ${ }^{11}$. L'année suivante, il confirme cette orientation avec la création d'une revue périodique consacrée à l'hygiène: Igea. Giornale d'igiene e medicina preventiva ${ }^{12}$. Dès lors, sa vocation de divulgateur scientifique ne fera que se renforcer : quatre ans plus tard, il inaugure la série (ininterrompue de 1866 à 1905) de l'Almanacco igienico popolare ${ }^{13}$, une collection économique de livres au format de poche, à périodicité annuelle, qui traitent de sujets de médecine de façon abordable ${ }^{14}$.

5 L'hygiène est le véritable cheval de bataille de Mantegazza. Sa conception de cette récente discipline médicale, dont le but est la prévention des maladies et la protection de la santé publique, est symptomatique de son attitude générale face à la vie : il la définit comme étant

toute une science sociale qui [...] puise sa lumière et sa force de la physiologie, et qui tend la main à l'économie politique et à la législation ${ }^{15}$.

C'est donc tout naturellement qu'il s'engage en politique : en 1865 il est élu député de la circonscription de Monza, sa ville natale, jusqu'à la défaite de la droite en 1876. Cette 
même année, il sera élu sénateur. Sa bataille pour l'hygiène ne se limite donc pas à un simple exercice de style, c'est un véritable engagement personnel, qui se joue également sur le terrain de l'action concrète. Au service de cette mission qu'il s'est fixée, Mantegazza est amené à multiplier les instruments de combat. Parallèlement à son - ou plutôt à ses activité(s) d'enseignant-chercheur, il est donc également homme politique, romancier, chroniqueur ${ }^{16}$, biographe ${ }^{17}$, éditeur, journaliste, directeur de collection, moraliste ${ }^{18}$.

7 Son activité la plus remarquable concerne le secteur de la divulgation scientifique, qui s'adresse à un public de profanes. Néanmoins, la plupart des œuvres de ce type délivrent moins des principes scientifiques que des principes de conduite. Il suffit d'ouvrir, par exemple, le Dizionario d'igiene per le famiglie (1881), écrit à quatre mains avec la romancière Neera, pour s'en convaincre. Paolo Mantegazza et Neera prônent ouvertement un retour aux valeurs conservatrices:

tous nos préceptes d'hygiène [...] visent à faire revenir les femmes aux anciennes traditions ${ }^{19}$.

L'apologie des vertus domestiques féminines est en effet l'un des pivots du programme moralisateur qui sous-tend une grande partie de la production pseudo-scientifique de Mantegazza. Il s'agit de combattre la corruption des mœurs, le nervosisme des femmes citadines ainsi que la mauvaise éducation qu'elles donnent à leur progéniture. Il s'agit également, à plus large échelle, de vanter la vertu de la résignation pour conserver l'ordre social établi. On peut lire ainsi à l'entrée " Maison » :

La maison peut être riche de tous les raffinements du luxe ou être très simple,

dépourvue de tapis et de rideaux; mais elle est toujours belle si c'est une maison où

l'on aime, où l'on travaille, et où l'on se repose en paix ${ }^{20}$.

9 Les auteurs tentent clairement de brimer les éventuelles velléités de rébellion à une condition défavorisée en prônant d'autres valeurs que l'argent et le pouvoir: la santé, l'amour, le travail.

De la même façon que les œuvres de divulgation se prévalent de l'autorité scientifique de l'auteur pour exprimer des idées étrangères à la science, les œuvres de pseudolittératurese présentent comme des œuvres littéraires mais poursuivent des visées d'ordre sanitaire. Ce qui ressort de ce tableau, c'est le souci constant d'œuvrer pour le bien public, en promouvant une hygiène quotidienne préventive à travers des conseils pratiques, simples et facilement applicables; par exemple, pratiquer de grandes respirations (la "gymnastique pulmonaire») ou nettoyer régulièrement la maison. Divertissement, orgueil, appât du gain et désir de gloire sont sans doute des motivations qui entrent en compte dans la pratique éditoriale de Mantegazza, mais elles sont superfétatoires par rapport à la volonté d'améliorer la santé publique. C'est également le cas des romans. En écrivant Un giorno a Madera, Mantegazza a certainement cédé au plaisir personnel du voyageur qui se remémore son séjour en terrain exotique, mais la motivation première et prédominante du livre est incontestablement la divulgation de principes prophylactiques.

11 Le narrateur est un jeune médecin de vingt-deux ans. Il s'embarque sur un paquebotposte à destination du Brésil et du Rio de la Plata ${ }^{21}$. Durant la traversée, il rencontre William, un jeune anglais avec qui il se lie d'amitié. Arrivés à destination, les deux hommes se séparent. William continue à parcourir le monde tandis que le narrateur s'installe en Amérique du sud. Ils restent en contact jusqu'à ce que le narrateur reçoive la dernière lettre de William, accompagnée d'un paquet de lettres que le jeune homme lui 
confie pour qu'il les publie. Il espère que, de cette façon, sa mésaventure sera utile à autrui. Les lettres retracent en effet l'histoire de l'amour impossible du jeune anglais et d'Emma. On y apprend que la jeune fille avait juré à son père de ne jamais se marier pour éviter de reproduire la tragique erreur de son père : les deux parents d'Emma ainsi que tous ses frères et sœurs étaient morts de tuberculose. La jeune fille elle-même était malade ${ }^{22}$. William avait cependant insisté pour qu'elle consulte trois illustres médecins. C'est l'un d'eux qui lui conseilla d'aller à Madère et d'avoir foi en sa guérison. La jeune fille meurt pourtant, heureuse d'avoir accompli son devoir et de n'avoir pas cédé à la tentation de l'amour.

Le roman se caractérise d'abord par le mélange. En premier lieu, le texte fictionnel, qui constitue $90 \%$ du livre, est assorti d'un péritexte, qui représente les $10 \%$ restant. Ce péritexte est composé d'une brève préface intitulée Due parole ai miei elettori di Monza [Deux mots à mes électeurs de Monza] faisant office de déclaration d'intention, et de plusieurs annexes situées en fin d'ouvrage. Quant à la fiction, elle se divise elle-même en deux parties bien différentes : dans la première partie, qui correspond aux chapitres I à $\mathrm{IV}$, le narrateur est intradiégétique; la deuxième partie, en revanche, est exclusivement épistolaire : c'est la correspondance de William et Emma. La première partie représente $15 \%$ du livre. Elle mêle les passages narratifs (la traversée, la rencontre du narrateur et de William) et les passages descriptifs (le chapitre II, intitulé lui aussi Un giorno a Madera, pendant l'escale, est prétexte à digression sur les paysages, les spécialités et les coutumes de Madère). La deuxième partie représente $75 \%$ du livre et relate la deuxième histoire, l'histoire principale, celle d'Emma et de son amour impossible.

Le livre ressortit à différents genres de littérature et pseudo-littérature par la coprésence de trois niveaux de lecture et, en quelque sorte, de trois genres. Outre le genre proprement romanesque qu'est la fiction, on distingue deux genres littéraires "d'importation": la littérature médicale et la littérature touristique. La fiction ne concerne que le texte; le genre touristique concerne plus spécifiquement le péritexte, tandis que le genre médical est omniprésent, bien que ce soit le seul à être presque tacite. Selon l'instance narrative qui est en jeu, les différents genres ou sous-genres qui coexistent se mélangent au sein de chaque catégorie textuelle. Par exemple, le récit de voyage est repérable dans la fiction, au chapitre II, et dans les annexes du péritexte. Procédons, par souci de clarté, en suivant les trois types énoncés ${ }^{23}$.

Nous avons vu que la fiction se rattache à la littérature épistolaire (au chapitre V) et au récit de voyage (au chapitre II). Elle a également partie liée avec le genre autobiographique et la littérature édifiante. Le début du roman repose sur des coordonnées étrangement proches de la vie de l'auteur. L'histoire se passe dans les années Cinquante, le narrateur est un jeune médecin italien de vingt-deux ans, il quitte l'Angleterre pour l'Amérique latine. C'est un autoportrait relativement fidèle de Mantegazza qui, une fois son diplôme de médecine en poche, en 1854, voyagea en Europe avant de partir pour Rio de la Plata ${ }^{24}$. Il avait alors vingt-trois ans. Par ailleurs, si l'on se place du point de vue de l'avant-texte, c'est-à-dire sur le plan des intentions de l'auteur, l'histoire d'Emma appartient indiscutablement à la littérature édifiante. Dans la préface, Mantegazza affirme vouloir faire «un livre utile et moral $»^{25}$. Il vise à constituer un exemplum, un modèle à suivre. Les lectrices sont invitées à agir comme l'héroïne, c'est-àdire à écouter non pas leur cœur mais leur devoir (civique). Le discours du père est volontairement virulent : 
j'étais malade, j'avais dans le sang le germe de la maladie qui me tue maintenant, et je l'ai transmise à mes enfants et je les ai tués. Je n'avais pas le droit de devenir père ${ }^{26}$.

le l'auteur est implicite, il se niche, en filigrane, dans les discours de différents personnages (notamment le père de Emma, Emma elle-même) et se dissimule sous une forme prosaïque, étrangère au jargon médical. Le genre ou sous-genre médical est également enchâssé dans la fiction: les lettres d'Emma contiennent différents discours médicaux rapportés, ceux des trois médecins réputés qu'elle consulte à Londres. Mais, contre toute attente, le portrait que Mantegazza dresse de ses confrères est un véritable éreintement : le premier est passionné de médecine mais il est incapable de prêter une oreille attentive à la parole du patient. Le second n'a d'yeux que pour les contradictions des différentes théories médicales: il est incapable de donner confiance au patient ${ }^{28}$. Le troisième, toutefois, est persuadé que la clef de la guérison est la foi dans la guérison. C'est lui qui conseille à Emma d'aller à Madère. Le dr. Haug est estimé par les personnages, mais l'auteur lui donne tacitement tort puisqu'Emma ne guérit pas ${ }^{29}$. Au niveau diégétique, le bon sens «populaire» du père d'Emma l'emporte sur les assertions des savants. Mantegazza se situe donc bien, en tant que romancier, sur le terrain glissant de l'opinion et des idées, et non sur celui de la science et des faits.

Enfin Un giorno a Madera tient également du guide touristique, par le récit exotique des premiers chapitres, notamment du deuxième, par le titre et, bien sûr, par la présence des quatre appendices de caractère purement informatif, mêlant prose et tableaux chiffrés. Le premier contient des informations historiques et géographiques sur Funchal et l'île de Madère, assorties d'un tableau de la situation actuelle de l'île (système politique, éducatif, démographie). Le deuxième porte sur le climat de Madère et le troisième sur les moyens de s'y rendre (Mantegazza fournit les tarifs, horaires et correspondances, et indique même le montant des pourboires à verser aux stewards). Cette troisième annexe s'achève sur une bibliographie des monographies consacrées à l'étude du climat de Madère et de son effet sur les maladies pulmonaires. La quatrième est en revanche uniquement constituée d'une bibliographie générale sur Madère.

18 Le roman affiche ici des visées purement pratiques: le livre se présente donc, pour le lecteur - ou plus exactement pour la lectrice, à la fois comme un divertissement, un modèle de comportement et un outil. Pour l'auteur, en revanche, le roman est un instrument de communication. A l'instar des autres ouvrages de (pseudo-)littérature et de divulgation que Mantegazza publie, Un giorno a Madera est destiné à un usage éphémère : les informations du péritexte, notamment les renseignements tariffaires relatifs aux moyens de transport pour se rendre à Madère, ne sont nécessairement valables que pendant un laps de temps assez court ${ }^{30}$. L'espérance de vie très réduite du livre montre, une fois encore, que la préoccupation principale de Mantegazza écrivain, qui est le progrès moral et social des Italiens, répond à une exigence de réalisation concrète et 
urgente, que l'on pourrait résumer dans la formule : «ici et aujourd'hui ». Le roman fait partie d'un programme de prévention sanitaire plus vaste qui doit porter ses fruits à l'échelle d'une vie humaine.

Un giorno a Madera est donc un livre d'un nouveau genre, représentatif de la société de consommation en cours de construction, car c'est un livre avec date de péremption. La littérature est un simple canal de diffusion d'idées et, à terme, un moyen d'action, une arme contre les maux de l'époque contemporaine ${ }^{31}$. Il ne faut pas confondre la "page d'hygiène de l'amour» de Mantegazza et la "page d'amour » de Zola ${ }^{32}$, où il est également question d'amour et de phtisie, mais où les intentions de l'auteur sont tout à fait différentes. Le but de Zola est artistique et sa culture scientifique, indispensable à la bonne compréhension (donc à la bonne reproduction) du réel, n'est qu'un outil. En revanche, chez Mantegazza la représentation fictionnelle est sous-tendue par la propagande. L'outil est la littérature et le but est la diffusion de l'idée soi-disant scientifique, qui est en réalité une doctrine. Le romancier naturaliste agit en savant tandis que le savant agit en moraliste ${ }^{33}$.

Les romans ultérieurs de Mantegazza confirmeront cette orientation liminaire. Si cette prose est une "prose d'idées" au sens où les idées à divulguer priment sur les fins esthétiques, peut-être faut-il plutôt parler de " proses d'idée » en raison de trois facteurs : l'hétérogénéité générique de ces œuvres «pseudo-littéraires» (qui incluent les différentes publications de divulgation et les romans, eux-mêmes hybrides) ; l'unicité de sa motivation (faire une littérature utile et bienfaitrice, au service du programme d'assainissement moral et physique de la population); enfin, le caractère spéculatif et idéologique de cette prose, qui fait qu'elle relève de la pensée et non des faits objectifs, positifs, de la pratique scientifique. Car, si le roman est instrumentalisé au profit de la prévention sanitaire, inversement la science est instrumentalisée au profit de la doctrine.

21 Si on ne peut pas en vouloir à Mantegazza d'avoir fondé son programme prophylactique sur un postulat scientifique erroné, selon lequel la tuberculose est transmissible par voie héréditaire, alors qu'elle transmissible par voie aérienne (le bacille responsable de la maladie n'ayant été identifié par Koch qu'en 1882), sa prise de position en faveur d'une doctrine eugénique, en revanche, semble bien étrange si l'on considère qu'il existait de nombreux cas de guérison. Mantegazza l'évoque lui-même dans les annexes : " plusieurs [tuberculeux] voient leur état s'améliorer et nombreux sont ceux qui guérissent $»^{34}$. La santé publique prime sur la santé individuelle : il faut préserver le corps social, quitte à ce que des vies soient inutilement sacrifiées. Cependant, on explique plus difficilement pourquoi, après la découverte de Koch, Mantegazza n'a pas jugé utile d'apporter un démenti à la thèse implicitement soutenue dans le roman. Il aurait pu le mettre en conformité avec les dernières avancées de la recherche, par exemple en conseillant plutôt à l'entourage d'un malade d'éviter le contact avec les postillons et crachats du tuberculeux, mais il ne l'a pas fait. Certes, la découverte de Koch n'a pas eu d'application thérapeutique significative avant la Première Guerre : fallait-il pour autant, de la part d'un savant, continuer de prescrire une méthode préventive aussi drastique que l'abstinence ? La responsabilité scientifique et morale de l'auteur, dont le roman a connu de multiples rééditions, n'était-elle pas en cause ${ }^{35}$ ? Ou le moraliste a-t-il pris le dessus sur le savant, estimant qu'une approximation scientifique porteuse d'une saine morale valait mieux qu'une vérité scientifique aux résultats incertains? Une chose est sûre : cet ultime élément nous confirme que le roman est bien le siège de l'opinion subjective, fût-elle contraire à la science. L'usage de genres hybrides, aptes à toucher un public de profanes, 
conduit donc Mantegazza à galvauder sa rigueur scientifique. Le caractère trompeur du titre du roman, qui veut être alléchant mais qui ne reflète qu'une part mineure du texte, est un exemple de cette corruption; l'obstination à défendre la thèse eugénique en dépit des cas de guérison recensés et, plus tard, de la découverte du bacille de Koch, en est une autre. En débordant du champ de ses compétences initiales, Paolo Mantegazza ne verse pas seulement dans l'idéologie (un pas qu'il franchit sciemment et ouvertement dès le début de sa carrière de divulgateur), il parvient également à renoncer aux principes fondateurs de la science qu'il idolâtre.

\section{NOTES}

1. Ainsi Walter Pasini donne-t-il à sa monographie consacrée à Mantegazza, qu'il qualifie de l'épithète humoristique de «tuttologo" [spécialiste en toute chose], le sous-titre Elogio dell'eclettismo [Eloge de l'éclectisme] (Walter PASINI, Paolo Mantegazza ovvero l'elogio dell'eclettismo, Rimini, Panozzo, 1999, p. 165). Hormis les publications strictement scientifiques, aucun texte de Mantegazza n'échappe à l'ambiguïté générique. Le livre India, par exemple, apparaît à Ernesto Citro comme la «fusione dell'abito dello scienziato con quello del letterato " [fusion de la casquette de l'homme de science et de celle de l'homme de lettres] («India » di Paolo Mantegazza, in «Esperienze Letterarie » n. 4, 2002, p. 60). Les traductions françaises sont nôtres hormis celles qui sont tirées de la Physiologie du plaisir.

2. Il n'existe qu'une seule traduction française du roman: Une journée à Madère, trad. Mme C. Thiry, Paris, C. Reinwald, 1882.

3. «la storia naturale dell'uomo » (Walter PASINI, cit., p. 198).

4. Il s'agit de la première Physiologie d'une série qui lui vaudra un succès de librairie et une renommée exceptionnels. Publiée l'année même de la soutenance chez l'éditeur milanais Bernardoni, la Fisiologia del piacere est republiée en 1859 puis, à partir des années Soixante, connaît de multiples rééditions à une cadence remarquablement soutenue. Une traduction française paraît en 1886.

5. En 1891 encore, il publiera un Saggio di una fisiologia del bello [Essai pour une philosophie du beau], non traduit en français.

6. En 1862 il y crée le premier laboratoire de Pathologie expérimentale d'Europe.

7. Cf. Degli innesti animali e della produzione artificiale delle cellule (1865) [Des greffes animales et de la production artificielle des cellules]. Les notes qui suivent (nn. 8-10 puis, infra, nn. 16-18) proposent également un titre pour chaque domaine d'intervention, mais ce ne sont que des exemples : la production de Mantegazza est bien plus vaste.

8. Cf. Istruzioni per lo studio della psicologia comparata (1873) [Instructions pour l'étude de la psychologie comparée], avec Giglioli et Letourneau.

9. Cf. Atlante della espressione del dolore (1876) [Atlas de l'expression de la douleur]. Dans sa thèse de médecine, Mantegazza propose déjà la création d'une nouvelle science, "l'édonologie » ou « science du plaisir» (Physiologie du plaisir, tr. fr. G. Combes de Lestrade, Paris, Reinwald, 1886, p. 369). Il est toutefois parfaitement conscient du caractère subjectif, donc insaisissable pour les instruments de la science expérimentale, de cette nouvelle «science ». Il écrit en effet dans les aphorismes qui concluent l'ouvrage: « Des mille éléments qui peuvent modifier le moment fugitif du plaisir, le plus puissant est le centre cérébral. La même sensation peut être voluptueuse ou 
déchirante suivant d'état du moi. Ce n'est donc pas un paradoxe, mais une vérité physiologique incontestable que de dire qu'il n'existe pas de plaisir qui soit essentiellement et nécessairement tel » (Ibidem, aphorismes V et VI, p. 370).

10. Cf. Carlo Darwin e il suo ultimo libro (1868) [Charles Darwin et son dernier livre].

11. [Le bien et le mal. Livre pour tous].

12. [Igea. Journal d'hygiène et de médecine préventive]. Igea change de nom en 1873 et devient $I l$ medico di casa [Le médecin de famille] pour mieux s'adapter à un public non averti. En 1886, il lance et dirige la Piccola Biblioteca del popolo italiano [Petite Bibliothèque du peuple italien] chez l'éditeur Barbera.

13. [Almanach hygiénique populaire].

14. Les livres paraissent d'abord chez l'éditeur milanais Brigola puis, à partir de 1884 , chez Dumolard. L'appellation almanach est due au fait qu'ils comportaient, dans les premières pages, un calendrier annuel.

15. "tutta una scienza sociale che [...] aspetta luce e forza dalla fisiologia, e stende la mano all'economia politica e alla legislazione » (Trattato di igiene (1864) [Traité d'hygiène], in Walter PASINI, cit., p. 93). Le premier hygiéniste était le français Alexandre-Jean-Baptiste ParentDuchâtelet (mort en 1836). Mantegazza lui rend hommage dans le premier numéro de Igea.

16. Il est l'auteur de plusieurs récits de voyage comme Un viaggio in Lapponia (1881) [Un voyage en Laponie].

17. Cf. La mia mamma, Laura Solera Mantegazza (1886) [Ma maman, Laura Solera Mantegazza].

18. Mantegazza écrit des essais de mœurs comme Pensieri sul matrimonio (1886) [Pensées sur le mariage] et des essais socio-politiques comme Ordine e libertà. Conversazioni di politica popolare (1864) [Ordre et liberté. Conversations de politique populaire].

19. «Tutti i nostri precetti d'igiene [...] tendono a ricondurre la donna alle tradizioni antiche » ( Nervosismo », Paolo MANTEGAZZA et NEERA, Dizionario d'igiene per le famiglie [Dictionnaire d'hygiène pour les familles], Milano, Libri Scheiwiller, 1985, p. 237). On trouve également un éloge des travaux d'aiguille, considérés comme l'apogée de l'hygiène féminine.

20. «La casa può essere ricca di tutte le raffinatezze del lusso e può essere semplicissima, nuda di tappeti e di cortine; ma è sempre bella la casa dove si ama, dove si lavora, e si riposa in pace » («Casa », Ibidem, p. 97). On peut à ce propos citer Paola Govoni qui écrit, au sujet de la littérature de divulgation : «se, da un lato, si incitava il «popolo» alla lettura e al riscatto, dall'altro s'imponevano forti limitazioni all'ascesa sociale derivante da una maggiore cultura » [si, d'un côté, on incitait le "peuple » à lire et à s'élever, d'un autre côté, on posait de fortes entraves à l'ascension sociale consécutive à une plus grande culture] (Paola GOVONI, Un pubblico per la scienza. La divulgazione scientifica nell'Italia in formazione, Roma, Carocci, 2002, p. 112).

21. Le Río de la Plata (que l'on peut traduire par Fleuve d'Argent en français) est l'estuaire formé par la confluence des rìos Paranà et Uruguay. Il sépare l'Argentine de l'Uruguay.

22. «Emma non può esser tua, nè d'altri; essa è legata da un santo giuramento a viver sola, $a$ morir sola. Il mio sangue, è maledetto, è sacrato fatalmente a spegnersi in sè stesso » [« Emma ne peut être à toi, ni à personne d'autre ; elle est liée par un serment sacré à vivre seule, à mourir seule. Mon sang est maudit, il est condamné fatalement à s'éteindre en lui-même »] (Un giorno a Madera. Una pagina dell'igiene d'amore, Sesto San Giovanni, Madella, 1913, p. 47).

23. Luca Clerici propose une autre taxinomie de l'œuvre, fondée sur les différents types de discours, et parle de texte "semi-letterario» [« semi-littéraire»] (cf. Luca CLERICI, "Paolo Mantegazza tra scienza e letteratura. Strategie rappresentative e scelte stilistiche in Un giorno a Madera », in Acme, vol. 42, 1989, pp. 11-43).

24. On doit à Mantegazza un ouvrage intitulé Rio de la Plata e Tenerife (1867).

25. " un libro utile e morale » (Un giorno a Madera, cit., p. 4).

26. « io era ammalato, aveva nel sangue il germe della malattia che ora mi uccide, e l'ho trasmessa ai miei figliuoli e li ho uccisi. Io non aveva il diritto di diventar padre » (Ibidem, p. 52). 
27. Robert Koch découvrit le bacille de la tuberculose en 1882 .

28. Le dr. B. est « la caricature du fanatisme » et le dr. T. « la caricature du doute ». Cf. Ibidem, pp. 64-75.

29. Le discours tacite de l'auteur prime donc sur le discours explicite des personnages de médecins. C'est le signe d'une forte empreinte autoriale et de la sujetion de la composante fictionnelle au primat didactique-(pseudo-)médical.

30. Pourtant, les données chiffrées des quatre annexes restent inchangées jusqu'à la mort de l'auteur, en 1910 : le texte de la troisième édition de 1871 est rigoureusement identique à celui de 1913. On remarque pourtant que la documentation scientifique était très à jour pour la première parution du livre : les références les plus récentes de la Bibliographie datent de 1862 ; quant aux données statistiques de la Nota A, elles s'étendent jusqu'à l'année 1859: Mantegazza recense 50.000 émigrants quittant Madère chaque année de 1854 à 1859 (cf. Un giorno a Madera, Milano, Bernardoni, 1871, p. 170 ; Un giorno a Madera, Sesto S. Giovanni, Madella, 1913, p. 129). Il est étonnant que Mantegazza n'ait pas jugé utile d'actualiser son texte en l'espace de plus de quarante ans, et ce en dépit de l'inscription "accuratamente riveduta dall'Autore » [" soigneusement revue par l'Auteur »] portée sur l'édition de 1906, la dernière édition du vivant de l'auteur. Cette négligence trahit sans doute le fait que, avec les années, les motivations d'ordre pécuniaire l'emportèrent parfois sur les principes scientifiques qui régissaient ses jeunes années de recherche expérimentale. L'absence totale de nouveauté substantielle des ouvrages de divulgation étaye d'ailleurs cette hypothèse. On lit dans une lettre que Mantegazza adresse à Neera pendant la préparation du Dizionario d'igiene per le famiglie : « io non posso che ripetermi e il lettore troverà in forma alfabetica ciò che ho scritto cento volte sotto forma di capitoli, di almanacchi etc.» [ «je ne peux que me répéter et le lecteur trouvera sous une forme alphabétique ce que j'ai déjà écrit cent fois sous forme de chapitres, d'almanachs, etc. »] (Antonia ARSLAN, Margherita GANAZZOLI, Neera e Paolo Mantegazza : storia di una collaborazione (con 32 lettere inedite), in « La Rassegna della letteratura italiana », n. 1-2, anno $87^{\circ}$, serie VIII, gennaio-agosto 1983, p. 110).

31. "Con la veste 'scientifica', Mantegazza divulgava il suo 'messaggio morale' " [ Sous l'apparence 'scientifique', Mantegazza divulguait son 'message moral' »] (Paola GOVONI, cit., p. 229). D'ailleurs, on peut lire dans l'Almanach consacré aux Accidenti della vita [Accidents de la vie] : «per essere perfettamente sano, conviene essere buono » [" pour être parfaitement sain, il faut être bon »] (Ibidem, p. 234).

32. Une page d'amour (1878) est le huitième volume de la série des Rougon-Macquart. Les frères Goncourt et Zola ont déjà lancé le nouveau roman naturaliste depuis trois ans (Germinie Lacerteux est de 1865, Thérèse Raquin de1867) lorsque Un giorno a Mantegazza paraît mais, avec Mantegazza, nous sommes loin de la poétique réaliste du naturalisme. L'auteur exploite à l'envi tous les procédés narratifs susceptibles d'émouvoir le lecteur pour le gagner à sa cause: par exemple le suspense, la fin fermée ou l'identification du lecteur aux héros.

33. «Da tale punto di vista Un giorno a Madera altro non è che la messa in scena di una massima e non di una storia, ovvero della conclusione del sillogismo sul quale Mantegazza fonda la ragione e insieme l'utilità del suo progetto romanzesco: chi è ammalato di tisi non deve aver figli. » [« De ce point de vue Un giorno a Madera n'est autre que la mise en scène d'une maxime et non d'une histoire, la mise en scène de la conclusion du syllogisme sur lequel Mantegazza fonde à la fois la raison et l'utilité de son projet romanesque : celui qui est atteint de tuberculose ne doit pas avoir d'enfant »] (Luca CLERICI, cit., p. 26).

34. "molti [tisici] migliorano e parecchi guariscono " (Nota B, «Il clima di Madera», Un giorno a Madera, cit.,p. 133). Quelques pages plus loin, il répète le même type d'affirmation (cf. Nota $C$, «Vie per recarsi a Madera", Ibidem, p. 136). Des cas de guérisons étaient d'ailleurs connus depuis longtemps, si l'on se fie aux affirmations d'Edwin Lee, antérieures de plus de dix ans à la parution du roman de Mantegazza : " pendant qu'il n'y a encore qu'une prédisposition à la maladie, ainsi 
que quand les organes ne sont lésés qu'à un léger degré, et quelquefois même lorsqu'elle est plus avancée, pourvu que les malades conservent assez de forces pour pouvoir sans grand inconvénient prendre l'exercice en plein air quand le temps le permet, nous pouvons raisonnablement espérer - en plaçant les malades dans des conditions hygiéniques favorables [...] - de les voir se rétablir plus souvent qu'ils n'ont fait jusqu'à ce jour " (Edwin LEE, Notices sur Hyères et Cannes, suivies d'Observations sur l'influence du climat dans la phthisie pulmonaire, Paris, Baillière, 1857, pp. 78-79). Les «conditions hygiéniques favorables» auxquelles Lee fait allusion incluent le climat doux des régions méditerranéennes.

35. Entre la date charnière de 1882 et la mort de l'auteur, en 1910, le roman connut dix-huit nouvelles éditions (chez Treves jusqu'en 1892, chez Bemporad ensuite). L'édition de 1896 est « riveduta dall'autore » [« revue par l'auteur »] et celle de 1899 porte la mention « accuratamente riveduta dall'autore» [«soigneusement revue par l'auteur»]. Pourtant, il n'y a aucune modification du texte. Cf. supra note 30 .

\section{RÉSUMÉS}

En 1868, à l'âge de trente-sept ans, l'anthropologue italien Paolo Mantegazza, auteur de nombreux ouvrages de divulgation scientifique, inaugure une nouvelle stratégie de communication avec la publication d'un roman, Un jour à Madère (Un giorno a Madera). En s'adressant au public plus restreint et plus cultivé des romans, Mantegazza tente d'élargir son audience à de nouvelles couches de la population. Cependant, ses motivations restent inchangées. Le genre pseudo-scientifique dissimulait des visées morales : la santé du corps étant liée à la santé morale, ses livres de divulgation véhiculaient essentiellement une ligne de conduite, un décalogue des bonnes mœurs. Avec le nouveau genre pseudo-romanesque, l'auteur dissimule sous la forme alléchante de la fiction narrative des visées médicales, plus précisément prophylactiques. Dans les deux cas, néanmoins, formes et savoirs sont instrumentalisés : la déontologie scientifique et l'esthétique du texte littéraire s'inclinent devant l'idéologie.

Nel 1868, il trentasettenne antropologo monzese Paolo Mantegazza, già autore di numerosi libri di divulgazione scientifica, inaugura con la pubblicazione di un romanzo, Un giorno a Madera, una nuova strategia di comunicazione. Rivolgendosi al pubblico dei romanzi, più limitato e più colto, Mantegazza cerca di allargare il proprio lettorato. Tuttavia le sue motivazioni rimangono incambiate. Il genere pseudo-scientifico dissimulava fini moralistici : essendo la salute del corpo legata alla salute morale, i libri di divulgazione trasmettevano essenzialmente un modus operandi, un decalogo dei buoni costumi. Con il nuovo genere pseudo-romanzesco, l'autore dissimula sotto la forma allettante della finzione narrativa fini medici, nella fattispecie profilattici. In ambedue i casi, però, forme e saperi vengono strumentalizzati : la deontologia scientifica e l'estetica del testo letterario cedono il passo all'ideologia.

\section{INDEX}

Index chronologique : XIXe siècle

Mots-clés : Mantegazza, italien, divulgation scientifique, hygiène, médecine 


\section{AUTEUR}

\section{EDWIGE COMOY FUSARO}

Maître de conférences à l'université de Nice. Ses recherches portent sur la littérature de l'Italie post-unitaire. Elle a publié La nevrosi tra medicina e letteratura. Approccio epistemologico alle malattie nervose nella narrativa italiana (1865-1922), préfacé par F. Livi, Firenze, Polistampa, « Biblioteca di Medicina e Storia », 2007, 463 p. Affiliée au CIRCLES (EA 3159), elle enseigne en Langue, Littérature et Civilisation Italiennes. 